\title{
COBORDISM INVARIANTS OF FOLD MAPS
}

\author{
BOLDIZSÁR KALMÁR \\ Dedicated to Professor Yoshifumi Ando on the occasion of his sixtieth birthday.
}

\begin{abstract}
This is a survey paper of author's results on cobordism groups and semigroups of fold maps and simple fold maps. The results include: establishing a relation between fold maps and immersions through geometrical invariants of cobordism classes of fold maps and simple fold maps in terms of immersions with prescribed normal bundles, detecting stable homotopy groups of spheres as direct summands of the cobordism semigroups of fold maps, Pontryagin-Thom type construction for -1 codimensional fold maps and estimations about the cobordism classes of manifolds which have fold maps into stably parallelizable manifolds. In the last section some of these results are extended and we show that our invariants also detect stable homotopy groups of the classifying spaces $B O(k)$ as direct summands of the cobordism semigroups of fold maps.
\end{abstract}

\section{INTRODUCTION}

Fold maps of $(n+q)$-dimensional manifolds into $n$-dimensional manifolds have the formula

$$
f\left(x_{1}, \ldots, x_{n+q}\right)=\left(x_{1}, \ldots, x_{n-1}, \pm x_{n}^{2} \pm \cdots \pm x_{n+q}^{2}\right)
$$

as a local form around each singular point, and the subset of the singular points in the source manifold is a $(q+1)$-codimensional submanifold (for results about fold maps, see, for example, $1,2,2,3,5,18,9,18,26,35,36$ ). If we restrict a fold map to the set of its singular points, then we obtain a codimension one immersion into the target manifold of the fold map. This immersion together with more detailed informations about the neighbourhood of the set of singular points in the source manifold can be used as a geometrical invariant (see Section 2) of fold cobordism classes (see Definition 1.1) of fold maps (for results about cobordisms of singular maps with completely different approach from our present paper, see, for example, 7. 11, 12, 21, 25, 34, 48 and the works of Ando, Sadykov, Szücs and the author in References). In this way we obtain a geometrical relation between fold maps and immersions with prescribed normal bundles via cobordisms. In [18 we showed that these invariants describe completely the cobordisms of simple fold maps of $(n+1)$-dimensional manifolds into $n$-dimensional manifolds and in [17] we showed that these invariants detect direct summands of the cobordism group of fold maps, namely stable homotopy groups of spheres. In this paper we extend the results of 17] and show by constructing fibrations of Morse functions over immersed manifolds

2000 Mathematics Subject Classification. Primary 57R45; Secondary 57R75, 57R42, 55Q45.

Key words and phrases. Fold singularity, fold map, immersion, cobordism, stable homotopy group.

The author was supported by Canon Foundation in Europe. 
similarly to 17. that these invariants also detect stable homotopy groups of the classifying spaces $B O(k)$ as direct summands of the cobordism semigroups of fold maps.

The paper is organized as follows. In Section 1 we give basic notations and definitions, in Section 2 we define cobordism invariants of fold maps, summerize our already existing results concerning these invariants and study the cobordism classes of manifolds which have fold maps into stably parallelizable manifolds. In Section 3 we extend the results of [17].

The author would like to thank the referee for his helpful comments, which improved the paper.

\section{Preliminaries}

1.1. Notations. In this paper the symbol " $\amalg$ " denotes the disjoint union, for any number $x$ the symbol " $\lfloor x\rfloor$ " denotes the greatest integer $i$ such that $i \leq x, \gamma^{1}$ denotes the universal line bundle over $\mathbb{R} P^{\infty}, \varepsilon_{X}^{1}$ denotes the trivial line bundle over the space $X, \varepsilon^{1}$ denotes the trivial line bundle over the point, and the symbols $\xi^{k}, \eta^{k}$, etc. usually denote $k$-dimensional real vector bundles. The symbols $\operatorname{det} \xi^{k}$ and $T \xi^{k}$ denote the determinant line bundle and the Thom space of the bundle $\xi^{k}$, respectively. The symbol $\operatorname{Imm}_{N}^{\xi^{k}}(n-k, k)$ denotes the cobordism group of $k$ codimensional immersions into an $n$-dimensional manifold $N$ whose normal bundles are induced from $\xi^{k}$ (this group is isomorphic to the group $\left\{\dot{N}, T \xi^{k}\right\}$, where $\dot{N}$ denotes the one point compactification of the manifold $N$ and the symbol $\{X, Y\}$ denotes the group of stable homotopy classes of continuous maps from the space $X$ to the space $Y)$. The symbol $\operatorname{Imm}_{N}(n-k, k)$ denotes the cobordism group $\operatorname{Imm}_{N}^{\gamma^{k}}(n-k, k)$ where $\gamma^{k}$ is the universal bundle for $k$-dimensional real vector bundles and $N$ is an $n$-dimensional manifold. The symbol $\pi_{n}^{s}(X)\left(\pi_{n}^{s}\right)$ denotes the $n$th stable homotopy group of the space $X$ (resp. spheres). The symbol "id ${ }_{A}$ " denotes the identity map of the space $A$. The symbol $\varepsilon$ denotes a small positive number. All manifolds and maps are smooth of class $C^{\infty}$.

1.2. Fold maps. Let $n \geq 1$ and $q>0$. Let $Q^{n+q}$ and $N^{n}$ be smooth manifolds of dimensions $n+q$ and $n$ respectively. Let $p \in Q^{n+q}$ be a singular point of a smooth map $f: Q^{n+q} \rightarrow N^{n}$. The smooth map $f$ has a fold singularity of index $\lambda$ at the singular point $p$ if we can write $f$ in some local coordinates around $p$ and $f(p)$ in the form

$$
f\left(x_{1}, \ldots, x_{n+q}\right)=\left(x_{1}, \ldots, x_{n-1},-x_{n}^{2}-\cdots-x_{n+\lambda-1}^{2}+x_{n+\lambda}^{2}+\cdots+x_{n+q}^{2}\right)
$$

for some $\lambda(0 \leq \lambda \leq q+1)$ (the index $\lambda$ is well-defined if we consider that $\lambda$ and $q+1-\lambda$ represent the same index).

A smooth map $f: Q^{n+q} \rightarrow N^{n}$ is called a fold map if $f$ has only fold singularities.

A smooth map $f: Q^{n+q} \rightarrow N^{n}$ has a definite fold singularity at a fold singularity $p \in Q^{n+q}$ if $\lambda=0$ or $\lambda=q+1$, otherwise $f$ has an indefinite fold singularity of index $\lambda$ at the fold singularity $p \in Q^{n+q}$.

Let $S_{\lambda}(f)$ denote the set of fold singularities of index $\lambda$ of $f$ in $Q^{n+q}$. Note that $S_{\lambda}(f)=S_{q+1-\lambda}(f)$. Let $S_{f}$ denote the set $\bigcup_{\lambda} S_{\lambda}(f)$.

Note that the set $S_{f}$ is an $(n-1)$-dimensional submanifold of the manifold $Q^{n+q}$.

Note that each connected component of the manifold $S_{f}$ has its own index $\lambda$ if we consider that $\lambda$ and $q+1-\lambda$ represent the same index. 
Note that for a fold map $f: Q^{n+q} \rightarrow N^{n}$ and for an index $\lambda(0 \leq \lambda \leq\lfloor q / 2\rfloor)$ the codimension one immersion $\left.f\right|_{S_{\lambda}(f)}: S_{\lambda}(f) \rightarrow N^{n}$ of the singular set of index $\lambda S_{\lambda}(f)$ has a canonical framing (i.e., trivialization of the normal bundle) by identifying canonically the set of fold singularities of index $\lambda$ of the map $f$ with the fold germ $\left(x_{1}, \ldots, x_{n+q}\right) \mapsto\left(x_{1}, \ldots, x_{n-1},-x_{n}^{2}-\cdots-x_{n+\lambda-1}^{2}+x_{n+\lambda}^{2}+\cdots+x_{n+q}^{2}\right)$, see, for example, 30.

If $f: Q^{n+q} \rightarrow N^{n}$ is a fold map in general position, then the map $f$ restricted to the singular set $S_{f}$ is a general positional codimension one immersion into the target manifold $N^{n}$.

Since every fold map is in general position after a small perturbation, and we study maps under the equivalence relation cobordism (see Definition 1.1), in this paper we can restrict ourselves to studying fold maps which are in general position. Without mentioning we suppose that a fold map $f$ is in general position.

\subsection{Equivalence relations of fold maps.}

Definition 1.1. (Cobordism) Two fold maps $f_{i}: Q_{i}^{n+q} \rightarrow N^{n}(i=0,1)$ of closed (oriented) $(n+q)$-dimensional manifolds $Q_{i}^{n+q}(i=0,1)$ into an $n$-dimensional manifold $N^{n}$ are (oriented) cobordant if

(a) there exists a fold map $F: X^{n+q+1} \rightarrow N^{n} \times[0,1]$ of a compact (oriented) $(n+q+1)$-dimensional manifold $X^{n+q+1}$,

(b) $\partial X^{n+q+1}=Q_{0}^{n+q} \amalg(-) Q_{1}^{n+q}$ and

(c) $\left.F\right|_{Q_{0}^{n+q} \times[0, \varepsilon)}=f_{0} \times \operatorname{id}_{[0, \varepsilon)}$ and $\left.F\right|_{Q_{1}^{n+q} \times(1-\varepsilon, 1]}=f_{1} \times \operatorname{id}_{(1-\varepsilon, 1]}$, where $Q_{0}^{n+q} \times$ $[0, \varepsilon)$ and $Q_{1}^{n+q} \times(1-\varepsilon, 1]$ are small collar neighbourhoods of $\partial X^{n+q+1}$ with the identifications $Q_{0}^{n+q}=Q_{0}^{n+q} \times\{0\}$ and $Q_{1}^{n+q}=Q_{1}^{n+q} \times\{1\}$.

We call the map $F$ a cobordism between $f_{0}$ and $f_{1}$.

This clearly defines an equivalence relation on the set of fold maps of closed (oriented) $(n+q)$-dimensional manifolds into an $n$-dimensional manifold $N^{n}$.

We denote the set of fold (oriented) cobordism classes of fold maps of closed (oriented) $(n+q)$-dimensional manifolds into an $n$-dimensional manifold $N^{n}$ (into the Euclidean space $\left.\mathbb{R}^{n}\right)$ by $\mathcal{C}_{o} b_{N, f}^{(O)}(n+q,-q)\left(\right.$ by $\left.\mathcal{C} o b_{f}^{(O)}(n+q,-q)\right)$. We note that we can define a commutative semigroup operation in the usual way on the set of cobordism classes $\mathcal{C} o b_{N, f}^{(O)}(n+q,-q)$ by the disjoint union. If the target manifold $N^{n}$ is the Euclidean space $\mathbb{R}^{n}$ (or more generally $N^{n}$ has the form $\mathbb{R}^{1} \times M^{n-1}$, for some $(n-1)$-dimensional manifold $\left.M^{n-1}\right)$, then the elements in the semigroup $\mathcal{C} o b_{N, f}^{(O)}(n+q,-q)$ have an inverse: namely compose them with a reflection in a hyperplane (in $\{0\} \times M^{n-1}$ in general, see [46]). Hence the semigroups $\mathcal{C}_{o b}^{(O)}(n+$ $q,-q)$ are in this case actually groups.

We can refine this equivalence relation by considering the singular fibers (see, for example, [23, 36, 37, 49]) of a fold map.

Definition 1.2. Let $\tau$ be a set of singular fibers. Two fold maps $f_{i}: Q_{i}^{n+q} \rightarrow N^{n}$ $(i=0,1)$ with singular fibers in the set $\tau$ of closed (oriented) $(n+q)$-dimensional manifolds $Q_{i}^{n+q}(i=0,1)$ into an $n$-dimensional manifold $N^{n}$ are (oriented) $\tau$ cobordant if they are (oriented) cobordant in the sense of Definition 1.1 by a fold map $F: X^{n+q+1} \rightarrow N^{n} \times[0,1]$ whose singular fibers are in the set $\tau$.

In this way we can obtain the notion of simple fold cobordism of simple fold maps, i.e., let $\tau$ be the set of all the singular fibers which have at most one singular point 
in each of their connected components. We denote the set of simple fold cobordism classes of simple fold maps of closed (oriented) $(n+q)$-dimensional manifolds $Q^{n+q}$ into an $n$-dimensional manifold $N^{n}$ by $\mathcal{C}_{o b} b_{N, s}^{(O)}(n+q,-q)$. For results about simple fold maps, see, for example, [18, 30, 31, 32, 33, 39, 50. For results about $\tau$ cobordisms of fold maps with other set $\tau$ of singular fibers, see [20, 38].

\section{CoBOrDism InVARIANTS OF FOLD MAPS}

2.1. Fold germs and bundles of germs. Let us define the fold germ

$$
g_{\lambda, q}:\left(\mathbb{R}^{q+1}, 0\right) \rightarrow(\mathbb{R}, 0)
$$

by

$$
g_{\lambda, q}\left(x_{1}, \ldots, x_{q+1}\right)=\left(-x_{1}^{2}-\cdots-x_{\lambda}^{2}+x_{1+\lambda}^{2}+\cdots+x_{1+q}^{2}\right)
$$

for some $q \geq 1$ and $0 \leq \lambda \leq\lfloor(q+1) / 2\rfloor$.

We say that a pair of diffeomorphism germs

$$
\left(\alpha:\left(\mathbb{R}^{q+1}, 0\right) \rightarrow\left(\mathbb{R}^{q+1}, 0\right), \beta:(\mathbb{R}, 0) \rightarrow(\mathbb{R}, 0)\right)
$$

is an automorphism of a fold germ $g_{\lambda, q}:\left(\mathbb{R}^{q+1}, 0\right) \rightarrow(\mathbb{R}, 0)$ if the equation $g_{\lambda, q} \circ \alpha=$ $\beta \circ g_{\lambda, q}$ holds. We will work with bundles whose fibers and structure groups are germs and groups of automorphisms of germs, respectively, see [13.

If we have a fold map $f: Q^{n+q} \rightarrow N^{n}$, then for each $\lambda(0 \leq \lambda \leq\lfloor(q+1) / 2\rfloor)$ we have a fold germ bundle $\xi_{\lambda}(f): E\left(\xi_{\lambda}(f)\right) \rightarrow S_{\lambda}(f)$ over the singular set of index $\lambda S_{\lambda}(f)$, i.e., the fiber of $\xi_{\lambda}(f)$ is the fold germ $g_{\lambda, q}$, and over the singular set $S_{\lambda}(f)$ we have an $\left(\mathbb{R}^{q+1}, 0\right)$ bundle denoted by $\xi_{\lambda}^{q+1}(f): E\left(\xi_{\lambda}^{q+1}(f)\right) \rightarrow S_{\lambda}(f)$ and an $(\mathbb{R}, 0)$ bundle denoted by $\eta_{\lambda}^{1}(f): E\left(\eta_{\lambda}^{1}(f)\right) \rightarrow S_{\lambda}(f)$ together with a fiberwise map $E\left(\xi_{\lambda}(f)\right): E\left(\xi_{\lambda}^{q+1}(f)\right) \rightarrow E\left(\eta_{\lambda}^{1}(f)\right)$ which is equivalent on each fiber to the fold germ $g_{\lambda, q}$. The base space of the fold germ bundle $\xi_{\lambda}(f)$ is the singular set of index $\lambda S_{\lambda}(f)$ and the total space of this bundle $\xi_{\lambda}(f)$ is the fiberwise map $E\left(\xi_{\lambda}(f)\right): E\left(\xi_{\lambda}^{q+1}(f)\right) \rightarrow E\left(\eta_{\lambda}^{1}(f)\right)$ between the total spaces of the bundles $\xi_{\lambda}^{q+1}(f)$ and $\eta_{\lambda}^{1}(f)$. We call the bundle $\eta_{\lambda}^{1}(f)$ the target of the fold germ bundle $\xi_{\lambda}(f)$.

By [13, 42, 47] this bundle $\xi_{\lambda}(f)$ is a locally trivial bundle in a sense with a fiber $g_{\lambda, q}$ and an appropriate group of automorphisms

$$
\left(\alpha:\left(\mathbb{R}^{q+1}, 0\right) \rightarrow\left(\mathbb{R}^{q+1}, 0\right), \beta:(\mathbb{R}, 0) \rightarrow(\mathbb{R}, 0)\right)
$$

as structure group. By [13, 47] this structure group can be reduced to a maximal compact subgroup, namely to the group $O(\lambda) \times O(q+1-\lambda)$ in the case of $0 \leq \lambda<$ $(q+1) / 2$ and the group generated by the group $O(\lambda) \times O(\lambda)$ and the transformation $T=\left(\begin{array}{cc}0 & I_{\lambda} \\ I_{\lambda} & 0\end{array}\right)$ in the case of $\lambda=(q+1) / 2$, see, for example, [30]. We denote this latter group by $\langle O(\lambda) \times O(\lambda), T\rangle$.

It follows that the targets of the universal fold germ bundles of index $\lambda(0 \leq$ $\lambda \leq\lfloor(q+1) / 2\rfloor)$ are the trivial line bundles $\eta_{\lambda, q}^{1}: \varepsilon^{1} \rightarrow B(O(\lambda) \times O(q+1-\lambda))$ for $\lambda \neq(q+1) / 2$ and the appropriate line bundle $\eta_{(q+1) / 2, q}^{1}: l^{1} \rightarrow B\langle O(\lambda) \times O(\lambda), T\rangle$ for $q$ odd.

For oriented manifolds $Q^{n+q}$ and $N^{n}$ we have the analogous statements if we consider the subgroup $S(O(\lambda) \times O(q+1-\lambda))$ of orientation preserving transformations of the group $O(\lambda) \times O(q+1-\lambda)$ and the trivial line bundle $\varepsilon^{1} \rightarrow$ $B S(O(\lambda) \times O(q+1-\lambda))$ in the case of $0 \leq \lambda<(q+1) / 2$, and the appropriate 
subgroup $R_{\langle O(\lambda) \times O(\lambda), T\rangle}$ of the group $\langle O(\lambda) \times O(\lambda), T\rangle$ and the corresponding line bundle $\tilde{l}^{1} \rightarrow B R_{\langle O(\lambda) \times O(\lambda), T\rangle}$ in the case of $\lambda=(q+1) / 2$.

2.2. Immersions with prescribed normal bundles. As an imitation of the method of lifting positive codimensional singular maps [46] we can construct homomorphisms

$$
\xi_{\lambda, q}^{N}: \mathcal{C o b}_{N, f}^{(O)}(n+q,-q) \rightarrow \operatorname{Imm}_{N}^{\varepsilon_{B(O(\lambda) \times O(q+1-\lambda))}^{1}}(n-1,1)
$$

for $0 \leq \lambda<(q+1) / 2$ and

$$
\xi_{(q+1) / 2, q}^{N}: \operatorname{Cob}_{N, f}^{(O)}(n+q,-q) \rightarrow \operatorname{Imm}_{N}^{l^{1}}(n-1,1)
$$

for $q$ odd by mapping a cobordism class of a fold map $f$ into the cobordism class of the immersion of its fold singular set of index $\lambda S_{\lambda}(f)$ with normal bundle induced from the target of the universal fold germ bundle of index $\lambda$. (In the case of oriented manifolds $Q^{n+q}$ and $N^{n}$, we have the analogous homomorphisms

$$
\xi_{\lambda, q}^{O, N}: \mathcal{C} o b_{N, f}^{O}(n+q,-q) \rightarrow \operatorname{Imm}_{N}^{\varepsilon_{B S(O(\lambda) \times O(q+1-\lambda))}^{1}}(n-1,1)
$$

for $0 \leq \lambda<(q+1) / 2$ and

$$
\xi_{(q+1) / 2, q}^{O, N}: \operatorname{Cob}_{N, f}^{O}(n+q,-q) \rightarrow \operatorname{Imm}_{N}^{\tilde{l}^{1}}(n-1,1)
$$

for $q$ odd as well.) We used these homomorphisms in [17, 18, 20, in order to describe cobordisms of fold maps.

Since the cobordism group of $k$-codimensional immersions into a manifold $N^{n}$ with normal bundle induced from a vector bundle $\xi^{k}$ is isomorphic to the group of stable homotopy classes $\left\{\dot{N}, T \xi^{k}\right\}$ [4], the homomorphisms $\xi_{\lambda, q}^{N}$ for $\lambda \neq(q+1) / 2$ and $\xi_{(q+1) / 2, q}^{N}$ for $q$ odd can be considered as homomorphisms into the groups $\left\{\dot{N}, T \varepsilon_{B(O(\lambda) \times O(q+1-\lambda))}^{1}\right\}$ and $\left\{\dot{N}, T l^{1}\right\}$, respectively. Without mentioning we identify the cobordism group of $k$-codimensional immersions into a manifold $N^{n}$ with normal bundle induced from a vector bundle $\xi^{k}$ with the group of stable homotopy classes $\left\{\dot{N}, T \xi^{k}\right\}$.

We remark that the group $\left\{\dot{N}, T \varepsilon_{B(O(\lambda) \times O(q+1-\lambda))}^{1}\right\}$ is equal to the group $\left\{\dot{N}, S^{1} \vee\right.$ $S B(O(\lambda) \times O(q+1-\lambda))\} \cong\left\{\dot{N}, S^{1}\right\} \oplus\{\dot{N}, S B(O(\lambda) \times O(q+1-\lambda))\}$, where "S" denotes suspension. Therefore the homomorphisms $\xi_{\lambda, q}^{N}(\lambda \neq(q+1) / 2)$ can be written in the forms

$$
\xi_{\lambda, q, 1}^{N} \oplus \xi_{\lambda, q, 2}^{N}: \mathcal{C o b}_{N, f}^{(O)}(n+q,-q) \rightarrow\left\{\dot{N}, S^{1}\right\} \oplus\{\dot{N}, S B(O(\lambda) \times O(q+1-\lambda))\}
$$

obviously. Note that the homomorphism $\xi_{\lambda, q, 1}^{N}$ maps the fold cobordism class of a fold map $f$ into the cobordism class of the framed immersion of the singular set of index $\lambda$ of the fold map $f(0 \leq \lambda<(q+1) / 2)$.

Note that $B(O(\lambda) \times O(q+1-\lambda))=B O(\lambda) \times B O(q+1-\lambda)$ and there exists a composition of bundle maps $\varepsilon_{B O(q+1-\lambda)}^{1} \rightarrow \varepsilon_{B(O(\lambda) \times O(q+1-\lambda))}^{1} \rightarrow \varepsilon_{B O(q+1-\lambda)}^{1}$ which is the identity map. Therefore the group $\{\dot{N}, S B O(q+1-\lambda)\}$ is a direct summand of the group $\{\dot{N}, S B(O(\lambda) \times O(q+1-\lambda))\}$.

Let $\varrho_{\lambda, q}^{N}: \operatorname{Imm}_{N}^{\varepsilon_{B(O(\lambda) \times O(q+1-\lambda))}^{1}}(n-1,1) \rightarrow \operatorname{Imm}_{N}^{\varepsilon_{B O(q+1-\lambda)}^{1}}(n-1,1)$ denote the natural forgetting homomorphism. Then we have weaker cobordism invariants

$$
\varrho_{\lambda, q}^{N} \circ \xi_{\lambda, q}^{N}: \operatorname{Cob}_{N, f}^{(O)}(n+q,-q) \rightarrow\left\{\dot{N}, S^{1}\right\} \oplus\{\dot{N}, S B O(q+1-\lambda)\}
$$


$(0 \leq \lambda<(q+1) / 2)$.

Let $\tilde{\theta}_{q}^{N}: \operatorname{Imm}_{N}^{l^{1}}(n-1,1) \rightarrow \operatorname{Imm}_{N}(n-1,1)$ be the natural forgetting homomorphism, where $\eta_{(q+1) / 2, q}^{1}: l^{1} \rightarrow B\langle O(\lambda) \times O(\lambda), T\rangle$ is the target of the universal fold germ bundle of index $(q+1) / 2$ for $q$ odd.

A result about these invariants, which we obtain similarly to [17, is the following.

Theorem 2.1. For $n \geq 1$, an $n$-dimensional manifold $N^{n}$ and $q>0$ the cobordism semigroup $\mathcal{C}_{0} b_{N, f}^{(O)}(n+q,-q)$ of fold maps of (oriented) $(n+q)$-dimensional manifolds into $N^{n}$ contains the direct sum of $\lfloor(q+1) / 2\rfloor$ copies of the group $\left\{\dot{N}, S^{1}\right\}$. The restriction of the homomorphism $\xi_{\lambda, q}^{N}$ to the $(\lambda+1)$-th copy of $\left\{\dot{N}, S^{1}\right\}$ is an isomorphism $(\lambda=0, \ldots,\lfloor(q-1) / 2\rfloor)$.

Theorem 2.2. For $n \geq 1$, an $n$-dimensional manifold $N^{n}, q>0, k \geq 1$ and $q=2 k-1$ the cobordism semigroup $\mathcal{C}_{0} b_{N, f}(n+q,-q)$ of fold maps of unoriented $(n+q)$-dimensional manifolds into $N^{n}$ contains the direct sum $\operatorname{Imm}_{N}(n-1,1) \oplus$ $\bigoplus_{\lambda=0}^{\lfloor(q-1) / 2\rfloor}\left\{\dot{N}, S^{1}\right\}$ as a direct summand. The direct summand $\operatorname{Imm}_{N}(n-1,1)$ is detected by the homomorphism $\tilde{\theta}_{q}^{N} \circ \xi_{(q+1) / 2, q}^{N}: \operatorname{Cob}_{N, f}(n+q,-q) \rightarrow \operatorname{Imm}_{N}(n-1,1)$, where $\tilde{\theta}_{q}^{N} \circ \xi_{(q+1) / 2, q}^{N}$ maps a fold cobordism class $[f]$ to the cobordism class of the immersion of the singular set of index $k$ of the fold map $f$.

Remark 2.3. For $q$ even, in Theorems 2.1 and 2.2 we could also chose the indeces $\lambda=1, \ldots,\lfloor(q+1) / 2\rfloor$ for the homomorphisms $\xi_{\lambda, q, 1}^{N}$ instead of the indeces $\lambda=$ $0, \ldots,\lfloor(q-1) / 2\rfloor$. The proof is similar to that of [17], details are left to the reader.

Another application of our invariants is the following result about simple fold maps, which we obtained in 18 .

Let

$\gamma_{n}^{N}: \operatorname{Imm}_{N}^{\varepsilon^{1}}(n-1,1) \oplus \operatorname{Imm}_{N}^{\varepsilon^{1} \times \gamma^{1}}(n-2,2) \rightarrow \operatorname{Imm}_{N}(n-1,1) \oplus \operatorname{Imm}_{N}^{\gamma^{1} \times \gamma^{1}}(n-2,2)$ denote the natural forgetting homomorphism and

$$
\phi_{n}^{N}: \mathcal{C}_{o b}^{O}{ }_{N, s}(n+1,-1) \rightarrow \mathcal{C}_{o} b_{N, f}^{O}(n+1,-1)
$$

denote the natural homomorphism which maps a simple fold cobordism class into its fold cobordism class.

When the codimension is equal to -1 and the target manifold $N^{n}$ is oriented, in [18] we defined a semigroup homomorphism

$$
\mathcal{I}_{N}: \mathcal{C}_{o b} b_{N, s}^{O}(n+1,-1) \rightarrow \operatorname{Imm}_{N}^{\varepsilon^{1}}(n-1,1) \oplus \operatorname{Imm}_{N}^{\varepsilon^{1} \times \gamma^{1}}(n-2,2),
$$

which is just an adaptation of our invariant $\xi_{1,1}^{N}$ to the case of simple fold maps of oriented manifolds into oriented manifolds and their oriented simple fold cobordisms.

In [18] we showed that there exists a homomorphism

$$
\theta_{n}^{N}: \operatorname{Imm}_{N}^{\operatorname{det}\left(\gamma^{1} \times \gamma^{1}\right)}(n-1,1) \rightarrow \operatorname{Imm}_{N}(n-1,1) \oplus \operatorname{Imm}_{N}^{\gamma^{1} \times \gamma^{1}}(n-2,2)
$$

such that the diagram

$$
\begin{gathered}
\mathcal{C} o b_{N, s}^{O}(n+1,-1) \stackrel{\mathcal{I}_{N}}{\longrightarrow} \operatorname{Imm}_{N}^{\varepsilon^{1}}(n-1,1) \oplus \operatorname{Imm}_{N}^{\varepsilon^{1} \times \gamma^{1}}(n-2,2) \\
\downarrow \phi_{n}^{N} \\
\mathcal{C o b}_{N, f}^{O}(n+1,-1) \stackrel{\theta_{n}^{N} \circ \xi_{1,1}^{N}}{\longrightarrow} \operatorname{Imm}_{N}(n-1,1) \oplus \operatorname{Imm}_{N}^{\gamma^{1} \times \gamma^{1}}(n-2,2) .
\end{gathered}
$$


commutes and we obtained the following.

Theorem 2.4. Let $N^{n}$ be an oriented manifold. Then, the semigroup homomorphism $\mathcal{I}_{N}$ is a semigroup isomorphism between the cobordism semigroup $\mathcal{C o b}_{N, s}^{O}(n+$ $1,-1)$ of simple fold maps and the group $\operatorname{Imm}_{N}^{\varepsilon^{1}}(n-1,1) \oplus \operatorname{Imm}_{N}^{\varepsilon^{1} \times \gamma^{1}}(n-2,2)$.

Let

$$
\gamma_{n, 1}^{N}: \operatorname{Imm}_{N}^{\varepsilon^{1}}(n-1,1) \rightarrow \operatorname{Imm}_{N}(n-1,1)
$$

and

$$
\gamma_{n, 2}^{N}: \operatorname{Imm}_{N}^{\varepsilon^{1} \times \gamma^{1}}(n-2,2) \rightarrow \operatorname{Imm}_{N}^{\gamma^{1} \times \gamma^{1}}(n-2,2) .
$$

denote the natural forgetting homomorphisms.

Let $\pi_{n, 2}^{N}: \operatorname{Cob}_{N, s}^{O}(n+1,-1) \rightarrow \operatorname{Imm}_{N}^{\varepsilon^{1} \times \gamma^{1}}(n-2,2)$ denote the composition of $\mathcal{I}_{N}$ with the projection to the second factor.

Theorem 2.5. If two simple fold cobordism classes $[f]$ and $[g]$ in $\mathcal{C o b}_{N, s}^{O}(n+1,-1)$ are mapped into distinct elements by the natural homomorphism $\gamma_{n, 2}^{N} \circ \pi_{n, 2}^{N}$, then $[f]$ and $[g]$ are not fold cobordant. If $\gamma_{n, 2}^{N}$ is injective, then so is $\phi_{n}^{N}$.

If there exists a fold map from a not null-cobordant $(n+1)$-dimensional manifold into $N^{n}$, then $\phi_{n}^{N}$ is not surjective.

2.3. Pontryagin-Thom type construction. In [19] among others we show the following, which is a negative codimensional analogue of the Pontryagin-Thom type construction for singular maps in positive codimension [25, 40, 41, 43, 44, 45].

Theorem 2.6. There is a Pontryagin-Thom type construction for -1 codimensional fold maps, i.e.,

(1) there exists a universal fold map $\xi_{-1}: U_{-1} \rightarrow \Gamma_{-1}$ such that for every -1 codimensional fold map $g: Q^{n+1} \rightarrow N^{n}$ there exists a commutative diagram

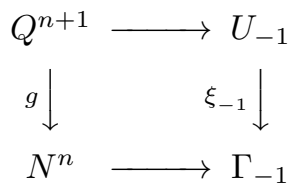

moreover the arising map $N^{n} \rightarrow \Gamma_{-1}$ is unique up to homotopy. It will be denoted by $\chi_{g}$. The space $\Gamma_{-1}$ is constructed by gluing together total spaces of vector bundles corresponding to the possible fold singular fibers and their automorphisms.

(2) For every positive integer $n$ and $n$-dimensional manifold $N^{n}$ there is a natural bijection

$$
\chi_{*}^{N}: \operatorname{Cob}_{N^{n}, f}(n+1,-1) \rightarrow\left[\dot{N}^{n}, \Gamma_{-1}\right]
$$

between the set of fold cobordism classes $\operatorname{Cob}_{N^{n}, f}(n+1,-1)$ and the set of homotopy classes $\left[\dot{N}^{n}, \Gamma_{-1}\right]$. The map $\chi_{*}^{N}$ maps a fold cobordism class $[g]$ into the homotopy class of the inducing map $\chi_{g}: \dot{N}^{n} \rightarrow \Gamma_{-1}$.

By Theorem 2.6 we have a bijective cobordism invariant $\chi_{*}^{N}: \operatorname{Cob}_{N^{n}, f}(n+$ $1,-1) \rightarrow\left[\dot{N}^{n}, \Gamma_{-1}\right]$ which is a group isomorphism $\chi_{*}^{\mathbb{R}^{n}}: \mathcal{C o b}_{f}(n+1,-1) \rightarrow \pi_{n}\left(\Gamma_{-1}\right)$ in the case of $N^{n}=\mathbb{R}^{n}$.

\footnotetext{
${ }^{1}$ The spaces $U_{-1}$ and $\Gamma_{-1}$ are not (finite dimensional) manifolds and so $\xi_{-1}$ is not a fold map.
} 
By defining the singular sets of index 0 and 1 of the universal fold map

$$
\xi_{-1}: U_{-1} \rightarrow \Gamma_{-1}
$$

in the obvious way and by inducing the immersions of these singular sets into the space $\Gamma_{-1}$ we get two representatives of two stable homotopy classes $\varrho_{0} \in$ $\left\{\Gamma_{-1}, T \varepsilon_{B O(2)}^{1}\right\}$ and $\varrho_{1} \in\left\{\Gamma_{-1}, T l^{1}\right\}$.

If we have a fold map $g: Q^{n+1} \rightarrow N^{n}$, then we have the stable homotopy class $\chi_{g}^{s}$ of the inducing map $\chi_{g}: \dot{N}^{n} \rightarrow \Gamma_{-1}$ in the group $\left\{\dot{N}^{n}, \Gamma_{-1}\right\}$. Hence we have the elements $\varrho_{0} \circ \chi_{g}^{s}$ and $\varrho_{1} \circ \chi_{g}^{s}$ in the groups $\left\{\dot{N}^{n}, T \varepsilon_{B O(2)}^{1}\right\}$ and $\left\{\dot{N}^{n}, T l^{1}\right\}$, respectively, which correspond to the elements $\xi_{0,1}^{N}([g])$ and $\xi_{1,1}^{N}([g])$, respectively.

Therefore we have the following.

Proposition 2.7. $\xi_{i, 1}^{N}([g])=\varrho_{i} \circ \chi_{g}^{s}$ for $i=0,1$.

2.4. Cobordism class of the source manifold of a fold map. We have a natural homomorphism $\sigma_{N, q}^{O}: \mathcal{C}_{o b}{ }_{N, f}(n+q,-q) \rightarrow \Omega_{n+q}$ which assigns to a class of a fold map $f: Q^{n+q} \rightarrow N^{n}$ the cobordism class $\left[Q^{n+q}\right]$ of the source manifold $Q^{n+q}$.

It is an easy fact that $\sigma_{\mathbb{R}, q}^{O}$ is surjective and the image of $\sigma_{\mathbb{R}^{2}, q}^{O}$ consists of the cobordism classes of $(2+q)$-dimensional manifolds with even Euler characteristic 22 .

Proposition 2.8. Let $N^{n}$ be a stably parallelizable n-dimensional manifold, where $n$ is even. Let $f: Q^{n+1} \rightarrow N^{n}$ be a fold map of an orientable manifold $Q^{n+1}$ such that its singular set $S_{f}$ is orientable. Then, the oriented cobordism class of the source manifold $Q^{n+1}$ is zero.

Proof. By 30, Lemma 3.1.] the bundle $T Q^{n+1} \oplus \varepsilon^{1}$ is isomorphic to the bundle $f^{*} T N^{n} \oplus \eta^{2}$ for some 2-dimensional bundle $\eta^{2}$. Hence the Stiefel-Whitney classes $w_{j}\left(Q^{n+1}\right)$ are zero for $j \geq 3$ and the Pontryagin classes $p_{j}\left(Q^{n+1}\right)$ are zero for $j \geq 2$. Since the Stiefel-Whitney class $w_{1}\left(Q^{n+1}\right)$ is zero and $n+1$ is odd, every StiefelWhitney and Pontryagin numbers of the manifold $Q^{n+1}$ are zero. This completes the proof.

Remark 2.9. Proposition 2.8 generalizes the analogous result about simple fold maps [18, 30.

Proposition 2.10. Let $q$ be even and let $N^{n}$ be a stably parallelizable manifold. Then, the rank of the image of $\sigma_{N, q}^{O}$ is less than or equal to the number of partitions of $(n+q) / 4$ where each number in a partition is less than or equal to $(q+1) / 2$. In particular, if $n>q+2$, then the homomorphism

$$
\sigma_{N, q}^{O} \otimes \mathbb{Q}: \mathcal{C}_{0} b_{N, f}^{O}(n+q,-q) \otimes \mathbb{Q} \rightarrow \Omega_{n+q} \otimes \mathbb{Q}
$$

is not surjective.

Proof. The statement follows from the fact that the possible non-zero Pontryagin numbers of the source manifold of a representative $f: Q^{n+q} \rightarrow N^{n}$ of an element in $\mathcal{C}_{o b} b_{N, f}^{O}(n+q,-q)$ are in bijection with the partitions of $(n+q) / 4$ where each number in a partition is less than or equal to $(q+1) / 2$. This holds because by [30, Lemma 3.1.] the bundle $T Q^{n+q} \oplus \varepsilon^{1}$ is isomorphic to the bundle $f^{*} T N^{n} \oplus \eta^{q+1}$ for some $(q+1)$-dimensional bundle $\eta^{q+1}$. Hence the Pontryagin classes $p_{i}\left(Q^{n+q}\right)$ are zero for $i>(q+1) / 2$. 
Corollary 2.11. Let $N^{n}$ be a stably parallelizable manifold.

(1) The orientable $(n+2)$-dimensional manifolds which have fold map into $N^{n}$ generate a subgroup of rank at most 1 in the cobordism group of $(n+2)$-dimensional manifolds.

(2) Let $n=4 k-2$. Let $Q^{4 k}$ be a (4k)-dimensional oriented manifold which has a fold map into the stably parallelizable manifold $N^{4 k-2}$. Then, the signature $\sigma\left(Q^{4 k}\right)$ of $Q^{4 k}$ is equal to $\frac{2^{2 k} B_{k}}{(2 k) !}(-1)^{k+1}\left\langle p_{1}^{k}\left(Q^{4 k}\right),\left[Q^{4 k}\right]\right\rangle$, where $B_{k}$ denotes the $k$ th Bernoulli number.

(3) Let $n=4 k-1$. If $Q^{4 k}$ has a fold map into $N^{4 k-1}$ such that the singular set $S_{f}$ is orientable, then the same holds for the signature of $Q^{4 k}$ as above.

Proof. (1) follows from Proposition 2.10. (2) and (3) follow from the Hirzebruch signature theorem (see, for example, [24, Lemma 19.1. and Theorem 19.4.]) and the fact that the Pontryagin classes $p_{i}\left(Q^{4 k}\right)$ are zero for $i>1$.

For other results about the signatures of source manifolds of fold maps, see, for example, [35, 37.

\section{Subgroups of the COBordism (SEMi)group of FOld MAPS}

In this section we extend the results of Theorems 2.1 and 2.2

Let $O(1, k)$ denote the subgroup of the orthogonal group $O(k+1)$ whose elements are of the form $\left(\begin{array}{cc}1 & 0 \\ 0 & M\end{array}\right)$ where $M$ is an element of the group $O(k)$.

Theorem 3.1. For $q>1$, the cobordism semigroup $\mathcal{C}_{0} b_{N, f}(n+q,-q)$ contains the direct sum

$$
\left\{\dot{N}, S^{1}\right\} \oplus\{\dot{N}, S B(O(1) \times O(q))\} \oplus \bigoplus_{2 \leq \lambda<(q+1) / 2}\left\{\dot{N}, S^{1}\right\} \oplus\{\dot{N}, S B O(q+1-\lambda)\}
$$

as a direct summand.

Proof. Similarly to [17] we construct fibrations of Morse functions of $(q+1)$-spheres

over the framed immersions in $\operatorname{Imm}_{N}^{\varepsilon_{B O(q+1-\lambda)}^{1}}(n-1,1) \cong\left\{\dot{N}, S^{1}\right\} \oplus\{\dot{N}, S B O(q+$ $1-\lambda)\}$ with the appropriate orthogonal group as symmetry group.

For $1 \leq j<(q+1) / 2$, let $h_{j}: S^{q+1} \rightarrow(-\varepsilon, \varepsilon)$ be a Morse function of the $(q+1)$ dimensional sphere into the open interval $(-\varepsilon, \varepsilon)$ with four critical points of index $0, j-1, j, q+1$, respectively, such that the critical value of index $j$ is zero in the interval $(-\varepsilon, \varepsilon)$.

In the following lemma we are looking for automorphisms of the Morse function $h_{j}$, i.e., auto-diffeomorphisms of the $(q+1)$-sphere which commute with the function $h_{j}(1 \leq j<(q+1) / 2)$.

Lemma 3.2. Let $1 \leq j<(q+1) / 2$. There exists an identification of the Morse function $h_{j}$ around its critical point of index $j$ with the fold germ

$$
g_{j, q}\left(x_{1}, \ldots, x_{q+1}\right)=\left(-x_{1}^{2}-\cdots-x_{j}^{2}+x_{1+j}^{2}+\cdots+x_{1+q}^{2}\right),
$$

such that under this identification

(1) any automorphism in the automorphism group $O(1) \times O(q)$ (in the case of $j=1$ )

(2) any automorphism in the automorphism group $O(1, j-1) \times O(q+1-j)$ (in the case of $j>1$ ) 
of the fold germ $g_{j, q}$ can be extended to an automorphism of the Morse function $h_{j}$.

Proof. Let $1 \leq j<(q+1) / 2$. Recall that the Morse function $h_{j}$ determines a handlebody decomposition of the $(q+1)$-sphere in the well-known way. We give a model of the $(q+1)$-sphere, its Morse function $h_{j}$ and its handlebody decomposition which shows the statement of the lemma.

First let us suppose that $j>1$. Let us identify the neighbourhood of the critical point of index 0 of $h_{j}$ with the hemisphere $D^{q+1} \subset S^{q+1}$ whose center is the critical point of index 0 . Let us identify the interior of the other hemisphere with the Euclidean space $\mathbb{R}^{q+1}$. We can choose this identification so that for the critical points of $h_{j}$ and the corresponding handles of the handlebody decomposition of the $(q+1)$-sphere we have the following.

(1) The critical point of index $j-1$ of $h_{j}$ corresponds to the point $(-1,0, \ldots, 0)$ in $\mathbb{R}^{q+1}$,

(2) the $(j-1)$-handle corresponds to a $(j-1)$-dimensional affine subspace $L^{j-1}$ in $\mathbb{R}^{q+1}$ which is orthogonal to the line $\left\langle x_{1}\right\rangle$ and contains the point $(-1,0, \ldots, 0)$,

(3) the critical point of index $j$ of $h_{j}$ corresponds to the origin in $\mathbb{R}^{q+1}$,

(4) the $j$-handle corresponds to the half space $H_{+}=\left\{\left(x_{1}, \ldots, x_{q+1}\right): x_{1} \geq\right.$ $-1\}$

(5) the critical point of index $q+1$ of $h_{j}$ corresponds to the point $(-2,0, \ldots, 0)$ in $\mathbb{R}^{q+1}$ and

(6) the $(q+1)$-handle corresponds to the half space $H_{-}=\left\{\left(x_{1}, \ldots, x_{q+1}\right)\right.$ : $\left.x_{1} \leq-1\right\}$.

Now we can see that the negative definite part of the fold germ $g_{j}$ corresponds to $J=\left(L^{j-1} \oplus\left\langle x_{1}\right\rangle\right) \cap H_{+}$and the positive definite part corresponds to the orthogonal complement of $J$, which lies in the hyperplane $H=\left\{x_{1}=0\right\}$. It is easy to check that an orthogonal transformation $O$ of $\mathbb{R}^{q+1}$ can be extended to an automorphism of the Morse function $h_{j}$ in this model if and only if the hyperplane $H$ and the affine subspace $L^{j-1}$ are invariant subspaces of the transformation $O$ and the line $\left\langle x_{1}\right\rangle$ is fixed pointwise. The statement of the lemma follows.

In the case of $j=1$ using the handle decomposition of the $(q+1)$-sphere determined by the Morse function $h_{1}$ it is easy to check that the claimed symmetries exist.

Let us define homomorphisms $\alpha_{1, q}^{N}: \operatorname{Imm}_{N}^{\varepsilon_{B(O(1) \times O(q))}^{1}}(n-1,1) \rightarrow \operatorname{Cob}_{N, f}(n+$ $q,-q)$ and $\alpha_{j, q}^{N}: \operatorname{Imm}_{N}^{\varepsilon_{B(O(1, j-1) \times O(q+1-j))}^{1}}(n-1,1) \rightarrow \operatorname{Cob}_{N, f}(n+q,-q)(2 \leq j<$ $(q+1) / 2)$ similarly to [17] as follows. Let $\left[g: M^{n-1} \rightarrow N^{n}\right]$ be an element of $\operatorname{Imm}_{N} \varepsilon_{B(O(1) \times O(q))}^{1}(n-1,1)$. Then the normal bundle of the immersion $g: M^{n-1} \rightarrow$ $N^{n}$ is induced from the trivial line bundle $\varepsilon_{B(O(1) \times O(q))}^{1}$. Let $\alpha_{1, q}^{N}([g])$ be the cobordism class of the fold map which we obtain by constructing the fibration of the Morse function $h_{1}$ over the manifold $M^{n-1}$ with the same symmetry group as that of the normal bundle of $g: M^{n-1} \rightarrow N^{n}$ induced from the bundle $\varepsilon_{B(O(1) \times O(q))}^{1}$, in a way analogous to the method described in [17]. By Lemma 3.2 the homomorphism $\alpha_{1, q}^{N}$ is well-defined. The definition of the homomorphisms $\alpha_{j, q}^{N}$ for $j>1$ is analogous.

Now we can see that the composition

$$
\xi_{1, q}^{N} \circ \alpha_{1, q}^{N}: \operatorname{Imm}_{N}^{\varepsilon_{B(O(1) \times O(q))}^{1}}(n-1,1) \rightarrow \operatorname{Imm}_{N}^{\varepsilon_{B(O(1) \times O(q))}^{1}}(n-1,1)
$$


is the identity map and the composition

$$
\varrho_{j, q}^{N} \circ \xi_{j, q}^{N} \circ \alpha_{j, q}^{N}: \operatorname{Imm}_{N}^{\varepsilon_{B(O(1, j-1) \times O(q+1-j))}^{1}}(n-1,1) \rightarrow \operatorname{Imm}_{N}^{\varepsilon_{B O(q+1-j)}^{1}}(n-1,1)
$$

restricted to the direct summand $\operatorname{Imm}_{N}^{\varepsilon_{B O(q+1-j)}^{1}}(n-1,1)$ of

$$
\operatorname{Imm}_{N}^{\varepsilon_{B(O(1, j-1) \times O(q+1-j))}^{1}}(n-1,1)
$$

is the identity map as well $(j<(q+1) / 2)$.

The remaining steps of the proof are obvious analogues of [17.

Remark 3.3. It follows that the composition

$$
\xi_{j, q}^{N} \circ \alpha_{j, q}^{N}: \operatorname{Imm}_{N}^{\varepsilon_{B(O(1, j-1) \times O(q+1-j))}^{1}}(n-1,1) \rightarrow \operatorname{Imm}_{N}^{\varepsilon_{B(O(j) \times O(q+1-j))}^{1}}(n-1,1)
$$

is equal to the natural homomorphism

$$
\begin{aligned}
\beta_{j, *}:\left\{\dot{N}, S^{1}\right\} \oplus\{\dot{N}, S B(O(1, j-1) & \times O(q+1-j))\} \longrightarrow \\
& \left\{\dot{N}, S^{1}\right\} \oplus\{\dot{N}, S B(O(j) \times O(q+1-j))\}
\end{aligned}
$$

induced by the map $\beta_{j}: B O(1, j-1) \rightarrow B O(j)(2 \leq j<(q+1) / 2)$. Therefore if the map $\beta_{j, *}$ is injective or an isomorphism, then the cobordism semigroup $\operatorname{Cob}_{N, f}(n+$ $q,-q)$ contains the group $\{\dot{N}, S B(O(1, j-1) \times O(q+1-j))\}$ as a subgroup or as a direct summand, respectively.

For example, in the case of $n=2$ and $N^{2}=\mathbb{R}^{2}$, we have that the cobordism group $\mathcal{C}_{o b}(2+q,-q)$ contains the direct sum

$$
\bigoplus_{1 \leq j<(q+1) / 2} \pi_{1}^{s} \oplus \pi_{1}^{s}(B(O(1, j-1) \times O(q+1-j)))=\mathbb{Z}_{2}^{3\lfloor q / 2\rfloor}
$$

as a direct summand, where $O(1,0)$ denotes the orthogonal group $O(1)$. Similarly, the cobordism group $\mathcal{C}_{o b} O(2+q,-q)$ contains the group $\mathbb{Z}_{2}^{2\lfloor q / 2\rfloor}$ as a direct summand.

\section{REFERENCES}

[1] Y. Ando, Fold-maps and the space of base point preserving maps of spheres, J. Math. Kyoto Univ. 41 (2002), 693-737.

[2] _ Invariants of fold-maps via stable homotopy groups, Publ. RIMS, Kyoto Univ. 38 (2002), 397-450.

[3] _ Existence theorems of fold-maps, Japan. J. Math. 30 (2004), 29-73.

[4] Cobordisms of maps without prescribed singularities, arXiv:math.GT/0412234 1 .

[5] - Stable homotopy groups of spheres and higher singularities, J. Math. Kyoto Univ. 46 (2006) 147-165.

[6] - Cobordisms of maps with singularities of a given class, arXiv:0707.4329v1.

[7] T. Ekholm, A. Szücs and T. Terpai, Cobordisms of fold maps and maps with prescribed number of cusps, arXiv:math.GT/0701433 1 .

[8] J. M. Eliashberg, On singularities of folding type, Math. USSR-Izv. 4 (1970), 1119-1134.

[9] , Surgery of singularities of smooth mappings, Math. USSR-Izv. 6 (1972), 13021326.

[10] M. Gromov, Stable mappings of foliations into manifolds, Math. USSR-Izv. 3 (1969), 671-694.

[11] K. Ikegami, Cobordism group of Morse functions on manifolds, Hiroshima Math. J. 34 (2004), 211-230.

[12] K. Ikegami and O. Saeki, Cobordism group of Morse functions on surfaces, J. Math. Soc. Japan 55 (2003), 1081-1094. 
[13] K. Jänich, Symmetry Properties of Singularities of $C^{\infty}$-Functions, Math. Ann. 238 (1978), $147-156$.

[14] B. Kalmár, Cobordism group of Morse functions on unoriented surfaces, Kyushu J. Math. 59 (2005), 351-363.

[15] Cobordism group of fold maps of oriented 3-manifolds into the plane, to appear.

[16] - Pontrjagin-Thom construction for singular maps with negative codimension, degree-thesis, 2005.

[17] $\_$Fold cobordisms and stable homotopy groups, to appear.

[18] . Fold maps and immersions from the viewpoint of cobordism, arXiv:0705.2731 v1.

[19] Pontryagin-Thom type construction for negative codimensional singular maps, arXiv:math.GT/0612116 1 .

[20] Cobordisms of fold maps with prescribed singular fibers, preprint in preparation.

[21] U. Koschorke, Vector fields and other vector bundle morphisms - a singularity approach, Lect. Notes in Math. 847, Springer-Verlag, 1981.

[22] H. Levine, Elimination of cusps, Topology 3 (1965) suppl. 2, 263-296.

[23] Classifying immersions into $\mathbb{R}^{4}$ over stable maps of 3-manifolds into $\mathbb{R}^{2}$, Lect. Notes in Math. 1157, Springer-Verlag, 1985.

[24] J. W. Milnor and J. D. Stasheff, Characteristic classes, Annals of Mathematics Studies, No. 76. Princeton University Press, Princeton, 1974.

[25] R. Rimányi and A. Szűcs, Generalized Pontrjagin-Thom construction for maps with singularities, Topology 37 (1998), 1177-1191.

[26] R. Sadykov, Elimination of singularities of smooth mappings of 4-manifolds into 3manifolds, Topology Appl. 144 (2004), no. 1-3, 173-199.

[27] , Bordism groups of special generic mappings, Proc. Amer. Math. Soc. 133 (2005), no. 3, 931-936.

[28] _ Bordism groups of solutions to differential relations, arXiv:math.AT/0608460 1 .

[29] Cobordism groups of Morin maps, preprint in preparation.

[30] O. Saeki, Notes on the topology of folds, J. Math. Soc. Japan 44 (1992), no. 3, 551-566.

[31] , Simple stable maps of 3-manifolds into surfaces. II, J. Fac. Sci. Univ. Tokyo Sect. IA Math. 40 (1993), no. 1, 73-124.

[32] Stable maps and links in 3-manifolds, Workshop on Geometry and Topology (Hanoi, 1993), Kodai Math. J. 17 (1994), no. 3, 518-529.

[33] 698.

[34] Cobordism groups of special generic functions and groups of homotopy spheres, Japan. J. Math. (N.S.) 28 (2002), no. 2, 287-297.

[35] _ Fold maps on 4-manifolds, Comment. Math. Helv. 78 (2003), no. 3, 627-647.

[36] - Topology of singular fibers of differentiable maps, Lect. Notes in Math. 1854, Springer-Verlag, 2004.

[37] O. Saeki and T. Yamamoto, Singular fibers of stable maps and signatures of 4-manifolds, Geom. Topol. 10 (2006), 359-399.

[38] Singular fibers and characteristic classes, preprint.

[39] K. Sakuma, On the topology of simple fold maps, Tokyo J. Math. 17 (1994), no. 1, 21-31.

[40] A. Szűcs, Analogue of the Thom space for mappings with singularity of type $\Sigma^{1}$ (in Russian), Math. Sb. (N.S.) 108 (150) (1979), 433-456, 478; English translation: Math. USSR-Sb. 36 (1980), 405-426.

[41] Cobordism groups of immersions with restricted self-intersection, Osaka J. Math. 21 (1984), 71-80.

[42] _ Universal Singular Map, Coll. Math. Soc. János Bolyai 55. Topology (Pécs, Hungary, 1989).

[43] , Topology of $\Sigma^{1,1}$-singular maps, Math. Proc. Camb. Phil. Soc. 121 (1997), 465477.

[44] - On the cobordism group of Morin maps, Acta Math. Hungar. 80 (1998), 191-209.

[45] _ Elimination of singularities by cobordism, Contemporary Mathematics 354 (2004), 301-324.

[46] - Cobordism of singular maps, arXiv:math.GT/0612152 v1.

[47] C. T. C. Wall, A second note on symmetry of singularities, Bull. London Math. Soc. 12 (1980), 347-354. 
[48] R. Wells, Cobordism of immersions, Topology 5 (1966), 281-294.

[49] T. Yamamoto, Classification of singular fibres of stable maps from 4-manifolds to 3manifolds and its applications, J. Math. Soc. Japan 58 (2006), No. 3, 721-742.

[50] Y. Yonebayashi, Note on simple stable maps of 3-manifolds into surfaces, Osaka J. Math. 36 (1999), no. 3, 685-709.

Kyushu University, Faculty of Mathematics, 6-10-1 Hakozaki, Higashi-Ku, Fukuoka 812-8581, JAPAN

E-mail address: kalmbold@yahoo.com 